\title{
圆渐开线变截面涡旋压缩机几何性能综合分析“
}

\author{
张朋成 彭 斌 张宇波 \\ (兰州理工大学机电工程学院 兰州 730050)
}

\begin{abstract}
摘要: 提出一种由不同基圆半径的圆渐开线组成的新型变截面浴旋压缩机型线, 组成形式为圆渐开线 I+圆渐开线 II +圆渐开 线 I。论述型线的生成方法, 给出型线的一般方程, 建立一系列圆渐开线变截面浴旋压缩机的几何模型。针对建立的几何模 型, 分析控制系数 $\theta 、 \varphi^{*} 、 R_{o r}$ 对变截面浴旋压缩机几何性能的影响。以圆渐开线 I 为基础, 构建圆渐开线 $\mathrm{I}+$ 高次曲线+圆 渐开线 I 的变截面浴旋压缩机的几何模型, 综合分析两类变截面浴旋压缩机的几何性能。结果表明: $\theta$ 取中值 $\theta_{M}, \varphi^{*}$ 取 $\varphi_{\text {end }}-2\left(n_{1}+1\right) \pi$, 控制系数 $R_{o r}$ 取较大值, 对应的几何性能较优。高次曲线变截面浴旋压缩机与中值 $\theta_{M}$ 对应的圆渐开线变截面 浴旋压缩机相似, 可相互替代。
\end{abstract}

关键词: 圆渐开线; 浴旋压缩机; 变截面; 几何模型; 控制系数

中图分类号: $\mathrm{TH} 45$

\section{Comprehensive Analysis of Geometric Performance of Circular Involute Variable Thickness Scroll Compressor}

\section{ZHANG Pengcheng PENG Bin ZHANG Yubo}

(School of Mechanical and Electronical Engineering, Lanzhou University of Technology, Lanzhou 730050)

\begin{abstract}
A new type of variable thickness scroll compressor line composed of circular involutes with different base circle radii is proposed, which is composed of a circular involute I+ circular involute II+ circular involute I. The method of generating the profile is discussed, the general equations of the profile are given, and a series of geometric models of the circular involute variable thickness scroll compressor are established. Aiming at the established geometric model, the influence of the control coefficients $\theta, \varphi^{*}$ and $R_{o r}$ on the geometric performance of the variable thickness scroll compressor is analyzed. Based on the circular involute I, a geometric model of variable thickness scroll compressors with circular involute I + higher-order curve + circular involute I is constructed, and the geometric performance of two types of variable thickness scroll compressors is comprehensively analyzed. The results show that: $\theta$ taking the median value $\theta_{M}, \varphi^{*}$ taking the value of $\varphi_{\text {end }}-2\left(n_{1}+1\right) \pi$, and $R_{o r}$ taking the larger, the corresponding geometric performance is better. Higher-order curve variable thickness scroll compressors are similar to the circular involute variable thickness scroll compressors corresponding to the median value $\theta_{M}$, and can be substituted for each other.
\end{abstract}

Key words: circular involute; scroll compressor; variable thickness; geometric model; control coefficient

\section{0 前言}

浴旋压缩机结构简单、效率高、噪声低、可靠 性高, 在制冷和空调、浴旋洜、涡旋增压器等领域 广泛应用 ${ }^{[1-4]}$ 。

目前, 国内外科研人员对涡旋压缩机的研究,

* 国家自然科学基金(51275226, 51675254，51966009)、国家重点研发 计划(SQ2020YFF0420989)和甘肃省陇原青年创新人才计划资助项目。 20191212 收到初稿, 20200825 收到修改稿
主要集中在等截面 ${ }^{[5]}$ 和变截面 ${ }^{[6]}$ 两个领域。等截面浴 旋压缩机由单一型线构成，如线段渐开线 ${ }^{[7]}$ 、圆渐 开线 ${ }^{[8-9]}$ 、正多边形渐开线 ${ }^{[10]}$ 等; 变截面浴旋压缩机 由组合型线构成, 构建方法通常以圆渐开线为基础, 另一曲线替代圆渐开线中间部分, 实现以较少圈数 达到同样的几何性能，如圆渐开线+圆弧+圆渐开 线 ${ }^{[1]}$ 、圆渐开线+高次曲线+圆弧 ${ }^{[12]}$ 等, 均由此方法 建立。变截面浴旋压缩机采用较少圈数即可实现高 压缩比、大排气量, 建立高效、实用、易加工的变 截面浴旋齿一直是研究的热点 ${ }^{[13]}$ 。BUSH 等首次提 
出由圆渐开线+高次曲线+圆弧构成的变截面浴旋 型线 ${ }^{[14]}$; 陈进等提出由圆渐开线+圆弧+圆渐开线构 成的变截面涡旋型线, 建立其几何模型 ${ }^{[15]}$; 彭斌等 提出由圆渐开线+高次曲线+圆渐开线的变截面浴 旋型线, 建立其几何模型 ${ }^{[16]}$; MURRAY 等对变截 面浴旋压缩机的结构进行了描述并对其性能的优势 做了分析 ${ }^{[17]}$ 。上述文献描述的变截面浴旋型线均由 2 种及 2 种以上组成, 在计算设计与加工制造等方 面较为复杂, 在一定程度上限制了变截面浴旋压缩 机的市场应用。

因此, 探究单一类型的型线是否可以构成变截 面浴旋压缩机型线, 有利于降低变截面浴旋压缩机 的设计与加工难度。本文提出一种由不同基圆半径 的圆渐开线组成的新型变截面浴旋压缩机型线, 型 线类型均为圆渐开线, 组成形式为圆渐开线 $I+$ 圆渐 开线 II+圆渐开线 I , 构建方法是首尾以半径为 $R_{1}$ 的圆渐开线为基础曲线, 中间段采用 $n_{2}$ 圈半径为 $R_{2}$ 的圆渐开线代替原 $n_{1}$ 圈半径为 $R_{1}$ 的圆渐开线部 分。针对建立的圆渐开线变截面涡旋压缩机几何模 型, 分析控制系数 $\theta 、 \varphi^{*} 、 R_{o r}$ 对圆渐开线变截面 浴旋压缩机几何性能的影响, 为开发高性能的浴旋 压缩机提供方向和借鉴。此外, 以圆渐开线 I 为基 础, 建立圆渐开线 I +高次曲线+圆渐开线 I 的高次 曲线变截面涡旋压缩机的几何模型, 综合分析两类 变截面浴旋压缩机的几何性能, 为变截面浴旋压缩 机的研究提供参考。

\section{1 几何模型的建立}

\section{1 基线方程}

圆渐开线 I 方程

$$
\left[\begin{array}{l}
X_{2} \\
Y_{2}
\end{array}\right]=R_{1}\left[\begin{array}{l}
(\cos \varphi+\varphi \sin \varphi) \\
(\sin \varphi-\varphi \cos \varphi)
\end{array}\right]
$$

式中, $\varphi \in\left[0, \varphi^{*}\right] \cup\left[\varphi^{*}+2 n_{1} \pi, \varphi_{\text {end }}\right] ; \varphi$ 为渐开线展角; $\varphi_{\text {end }}$ 为终端渐开线展角; $\varphi^{*}$ 为圆渐开线 I 第一段的 终端渐开线展开角; $n_{1}$ 为正整数; $R_{1}$ 为基圆半径。

圆渐开线 II 方程

$$
\left[\begin{array}{l}
X_{2} \\
Y_{2}
\end{array}\right]=R_{2}\left[\begin{array}{l}
(\cos \varphi+\varphi \sin \varphi) \\
(\sin \varphi-\varphi \cos \varphi)
\end{array}\right]
$$

式中, $\varphi \in\left[\varphi^{*}-\theta, \varphi^{*}-\theta+2 n_{2} \pi\right] ; \theta$ 为圆渐开线 I 第一段的终端渐开线展开角与圆渐开线 II 的开始渐 开线展开角的差值; $n_{2}$ 为正整数且满足 $n_{1}>n_{2} ; R_{2}$ 为基圆半径且满足 $R_{2} / R_{1}=n_{1} / n_{2}$ 。

圆渐开线 II (图 1 中 $S_{2}$ ) 绕基圆圆心逆时针旋转 $\theta$ 或顺时针旋转 $2 \pi-\theta$, 得到曲线 $S_{2}{ }^{\prime}$; 曲线 $S_{2}{ }^{\prime}$ 再 平移, 得到曲线 $S_{2}{ }^{\prime \prime}$; 曲线 $S_{2}{ }^{\prime \prime}$ 与圆渐开线 I (图 1 中 $S_{1}$ )构成基线。

能正确生成内外壁曲线的取值条件

$$
\left(\theta_{M}-\Delta \theta / 2\right)<\theta<\left(\theta_{M}+\Delta \theta / 2\right)
$$

式中, $\Delta \theta$ 为取值范围, 其大小与 $R_{1} / R_{2} 、 \varphi^{*} 、 R_{o r}$ 的取值有关; $\theta_{M}$ 为中值, 计算公式为

$$
\theta_{M}=\frac{\left(R_{2}-R_{1}\right)}{R_{2}} \varphi^{*}
$$

根据 $\theta$ 取值的变化, 可以生成一系列基线。以 表 1 参数值作图, 基线生成过程如图 1 所示。

表 1 基本参数值

\begin{tabular}{cc}
\hline 参数 & 数值 \\
\hline 基圆半径 $R_{1} / \mathrm{mm}$ & 10 \\
基圆半径 $R_{2} / \mathrm{mm}$ & 20 \\
圈数 $n_{1}$ & 2 \\
圈数 $n_{2}$ & 1 \\
渐开角 $\varphi^{*} / \mathrm{rad}$ & $2 \pi$ \\
终端渐开角 $\varphi_{\text {end }} / \mathrm{rad}$ & $8 \pi$ \\
中值 $\theta_{M} / \mathrm{rad}$ & $\pi$ \\
回转半径 $R_{o r} / \mathrm{mm}$ & $5 \pi$ \\
\hline
\end{tabular}

变换后的圆渐开线 II 方程

$$
\left[\begin{array}{l}
X \\
Y
\end{array}\right]=R_{2}\left[\begin{array}{l}
\cos \varphi+(\varphi-\theta) \sin \varphi \\
\sin \varphi-(\varphi-\theta) \cos \varphi
\end{array}\right]-\left[\begin{array}{l}
\Delta x \\
\Delta y
\end{array}\right]
$$

式中, $\varphi \in\left[\varphi^{*}-\theta, \varphi^{*}-\theta+2 n_{2} \pi\right] ; \Delta x 、 \Delta y$ 为坐标差 值, 计算公式如下

$$
\left[\begin{array}{c}
\Delta x \\
\Delta y
\end{array}\right]=\left[\begin{array}{c}
{\left[\left(R_{2}-R_{1}\right) \cos \varphi^{*}+\left(R_{2}-R_{1}\right) \times\right.} \\
\left.\varphi_{1} \sin \varphi^{*}-R_{2} \theta \sin \varphi^{*}\right] \\
{\left[\left(R_{2}-R_{1}\right) \sin \varphi^{*}-\left(R_{2}-R_{1}\right) \times\right.} \\
\left.\varphi_{1} \cos \varphi^{*}+R_{2} \theta \cos \varphi^{*}\right]
\end{array}\right]
$$

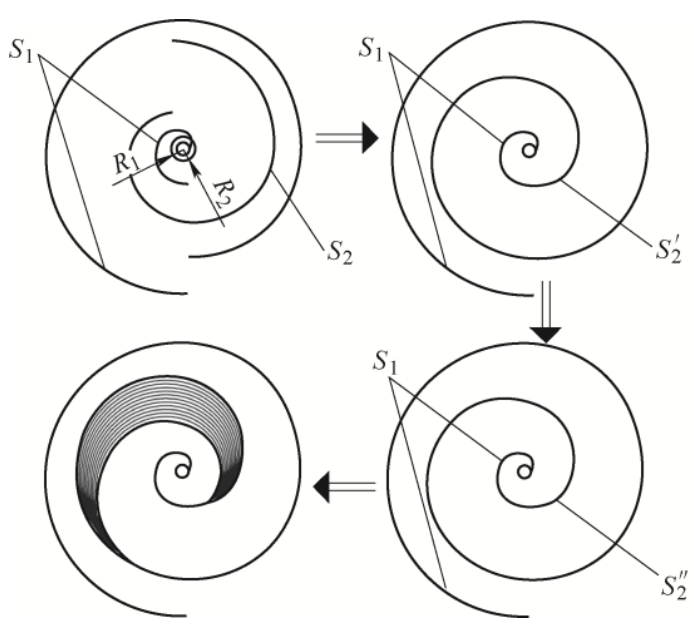

图 1 基线生成示意图 


\section{2 涡旋齿内外壁生成}

基线向外法向等距 ${ }^{[18]} 0.5 R_{o r}$, 确定静浴旋齿的 内壁 $\left(S_{a b}+S_{b c}+S_{c d}\right)$, 向内等距 $0.5 R_{o r}$, 确定动涡旋齿 的外壁 $\left(S_{e f}+S_{f g}+S_{g h}\right)$; 将前述所得的静浴旋齿内壁 $\left(S_{a b}+S_{b c}+S_{c d}\right)$ 、动浴旋齿外壁 $\left(S_{e f}+S_{f g}+S_{g h}\right)$ 分别 绕基圆圆心旋转 $\pi$, 得到动涡旋齿内壁 $\left(S_{a b}{ }^{\prime}+S_{b c}{ }^{\prime}+S_{c d}{ }^{\prime}\right)$ 和静浴旋齿外壁 $\left(S_{e f}{ }^{\prime}+S_{f g}{ }^{\prime}+S_{g h}{ }^{\prime}\right)$ 。

删除动静盘内壁最外圈多余的 $1 / 2$ 圈曲线, 得到静 涡旋齿内壁 $\left(S_{a b}+S_{b c}+S_{c i}\right)$ 和动涡旋齿内壁 $\left(S_{a b}{ }^{\prime}+S_{b c}{ }^{\prime}+S_{c i}{ }^{\prime}\right)$; 分别与静浴旋齿外壁 $\left(S_{e f}{ }^{\prime}+S_{f g}{ }^{\prime}+S_{g h}{ }^{\prime}\right)$ 和动浴旋齿外壁 $\left(S_{e f}+S_{f g}+S_{g h}\right)$ 构 成完整的动静浴旋齿。以表 1 参数作图, 生成的浴 旋齿内外壁如图 2 所示。

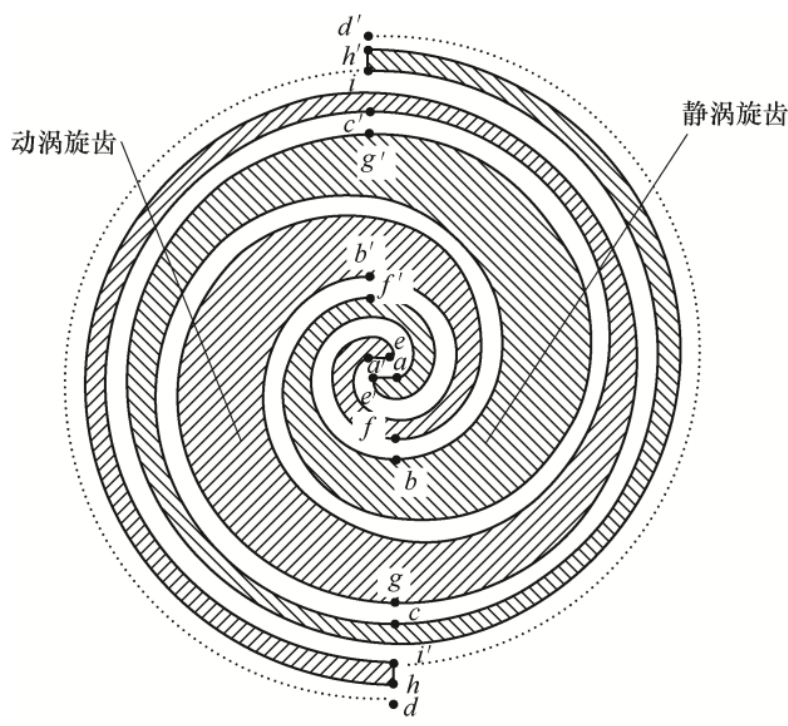

图 2 浴旋齿内外壁示意图

浴旋齿内壁方程如下。

第一段 $S_{a b}\left(S_{a^{\prime} b^{\prime}}\right)$ 方程

$$
\left[\begin{array}{l}
X \\
Y
\end{array}\right]=R_{1}\left[\begin{array}{l}
(\cos \varphi+\varphi \sin \varphi) \\
(\sin \varphi-\varphi \cos \varphi)
\end{array}\right]+\left[\begin{array}{l}
0.5 R_{o r} \sin \varphi \\
-0.5 R_{o r} \cos \varphi
\end{array}\right]
$$

式中, $\varphi \in\left[0, \varphi^{*}\right]$ 。

第二段 $S_{b c}\left(S_{b^{\prime} c^{\prime}}\right)$ 方程

$$
\begin{aligned}
& {\left[\begin{array}{l}
X \\
Y
\end{array}\right]=R_{2}\left[\begin{array}{l}
\cos \varphi+(\varphi-\theta) \sin \varphi \\
\sin \varphi-(\varphi-\theta) \cos \varphi
\end{array}\right]} \\
& -\left[\begin{array}{l}
\Delta x \\
\Delta y
\end{array}\right]+\left[\begin{array}{l}
0.5 R_{o r} \sin \varphi \\
-0.5 R_{o r} \cos \varphi
\end{array}\right]
\end{aligned}
$$

式中, $\varphi \in\left[\varphi^{*}-\theta, \varphi^{*}-\theta+2 n_{2} \pi\right]$ 。

第三段 $S_{c i}\left(S_{c^{\prime} i^{\prime}}\right)$ 方程

$$
\left[\begin{array}{l}
X \\
Y
\end{array}\right]=R_{1}\left[\begin{array}{c}
(\cos \varphi+\varphi \sin \varphi) \\
(\sin \varphi-\varphi \cos \varphi)
\end{array}\right]-\left[\begin{array}{l}
0.5 R_{o r} \sin \varphi \\
-0.5 R_{o r} \cos \varphi
\end{array}\right]
$$

式中, $\varphi \in\left[\varphi^{*}+2 n_{1} \pi, \varphi_{\text {end }}-\pi\right]$ 。

涡旋齿外壁方程

第一段 $S_{e f}\left(S_{e^{\prime} f^{\prime}}\right)$ 方程

$$
\left[\begin{array}{l}
X \\
Y
\end{array}\right]=R_{1}\left[\begin{array}{l}
(\cos \varphi+\varphi \sin \varphi) \\
(\sin \varphi-\varphi \cos \varphi)
\end{array}\right]-\left[\begin{array}{l}
0.5 R_{o r} \sin \varphi \\
-0.5 R_{o r} \cos \varphi
\end{array}\right]
$$

式中, $\varphi \in\left[0, \varphi^{*}\right]$ 。

第二段 $S_{f g}\left(S_{f g^{\prime}}\right)$ 方程

$$
\begin{aligned}
& {\left[\begin{array}{l}
X \\
Y
\end{array}\right]=R_{2}\left[\begin{array}{l}
\cos \varphi+(\varphi-\theta) \sin \varphi \\
\sin \varphi-(\varphi-\theta) \cos \varphi
\end{array}\right]-} \\
& {\left[\begin{array}{l}
\Delta x \\
\Delta y
\end{array}\right]-\left[\begin{array}{l}
0.5 R_{o r} \sin \varphi \\
-0.5 R_{o r} \cos \varphi
\end{array}\right]}
\end{aligned}
$$

式中, $\varphi \in\left[\varphi^{*}-\theta, \varphi^{*}-\theta+2 n_{2} \pi\right]$ 。

第三段 $S_{g h}\left(S_{g^{\prime} h^{\prime}}\right)$ 方程

$\left[\begin{array}{l}X \\ Y\end{array}\right]=R_{1}\left[\begin{array}{c}(\cos \varphi+\varphi \sin \varphi) \\ (\sin \varphi-\varphi \cos \varphi)\end{array}\right]-\left[\begin{array}{l}0.5 R_{o r} \sin \varphi \\ -0.5 R_{o r} \cos \varphi\end{array}\right]$

式中, $\varphi \in\left[\varphi^{*}+2 n_{1} \pi, \varphi_{\text {end }}\right]$ 。

为了得到更好的性能, 通常对浴旋齿齿头部分 进行修正, 修正方法有双圆弧修正 ${ }^{[19-20]}$ 、双圆弧加 直线修正 ${ }^{[21-22]}$ 和对称圆弧修正 ${ }^{[23]}$ 等。本文采用双圆 弧加直线修正, 动静浴旋齿基圆中心相距 $R_{o r}$ 进行 安装, 齿头修正及安装效果如图 3 所示, 齿头修正 前后浴旋齿工作腔投影面积变化如图 4 所示。

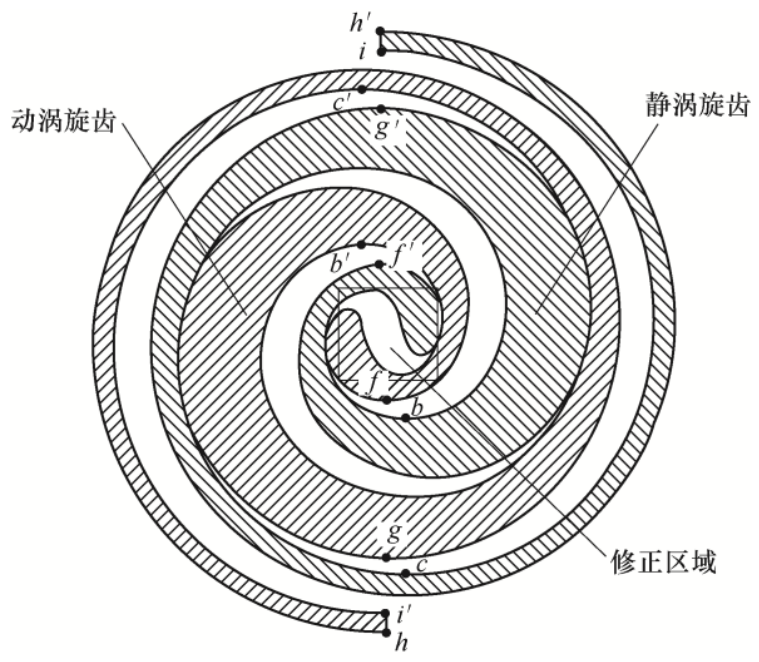

图 3 齿头修正及安装效果示意图

从图 4 可以看出：齿头修正后，压缩比更高、 排气过程更快，几何性能更优。

由式(1) (12)可知, 随着控制系数 $\theta 、 \varphi^{*} 、 R_{o r}$ 取值的变化, 生成的基线随之变化, 相应地所生成 的动静浴旋齿也随之变化。因此, 有必要分析控制 系数 $\theta 、 \varphi^{*} 、 R_{o r}$ 对几何性能的影响。 


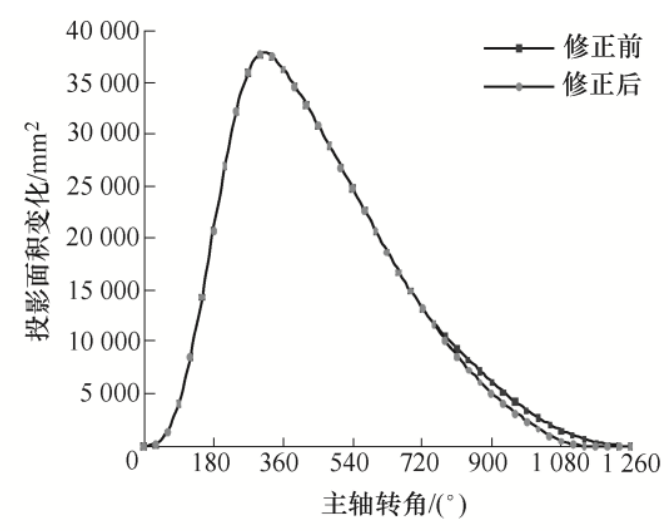

图 4 齿头修正前后工作腔投影面积对比

\section{2 控制系数 $\theta$ 对几何性能的影响}

其他参数保持不变, 仅改变控制系数 $\theta$, 研究 $\theta$ 取值的变化对几何性能的影响。

以表 1 参数值作图, 仅改变 $\theta$ 的取值, 其它参 数不变, $\theta$ 的取值如表 2 所示, 生成的一系列基线 如图 5a 所示。

表 $2 \theta$ 取值表

\begin{tabular}{cc}
\hline 渐开线差值 $\theta$ & 数值 \\
\hline 数值 1 & $0.7 \pi$ \\
数值 2 & $0.8 \pi$ \\
数值 3 & $0.9 \pi$ \\
数值 4 & $\pi$ \\
数值 5 & \\
数值 6 & $1.1 \pi$ \\
数值 7 & $1.2 \pi$ \\
\hline
\end{tabular}

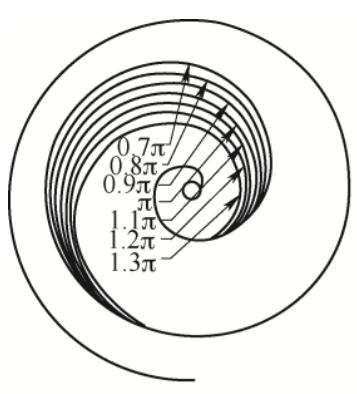

(a)

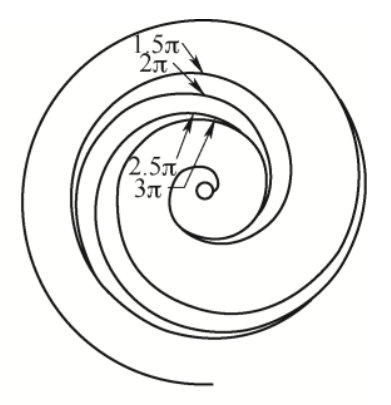

(b)
图 5 基线示意图

\section{1 控制系数 $\theta$ 对涡旋齿壁厚 $t$ 的影响}

以表 1 、表 2 参数值作图, 当 $\theta$ 取 $0.7 \pi$ 和 $1.3 \pi$ 生 成的内外壁发生相交, 相交处分别如图 6 中 $D_{1} 、 D_{2}$ 所示, 不能正确生成内外壁。其他取值均能正确生成 内外壁, 生成的壁厚 $t$ 随渐开线展角变化如图 7 所示。

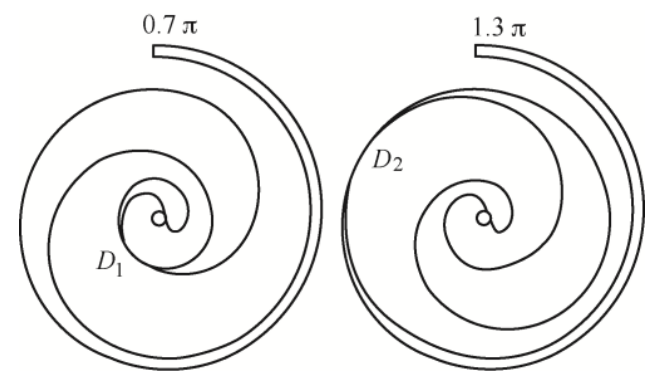

图 6 内外壁相交示意图

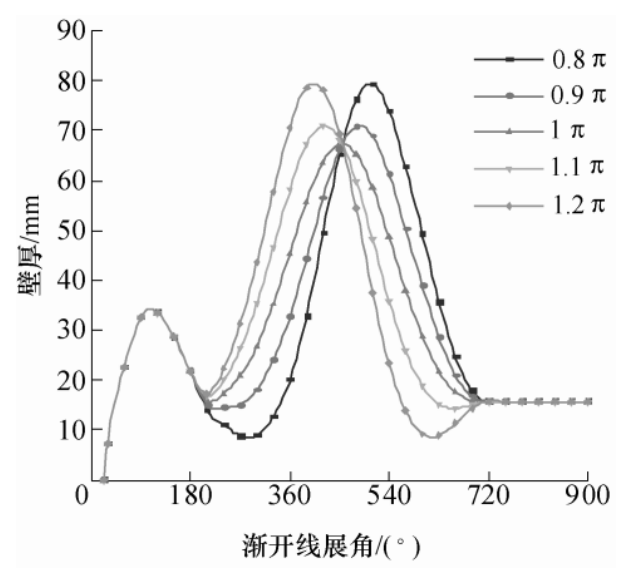

图 7 壁厚随渐开线展角的变化

从图 7 可知: $\varphi$ 小于 $180^{\circ}$ 时, 为齿头修正部分, 其壁厚先增大后减小, 因齿头修正非本文研究重点, 不做分析。 $\theta<\theta_{M}$ (图中 $0.7 \pi 、 0.8 \pi$ ), 壁厚 $t$ 随着渐 开线展角变化依次为减小、增大、再减小、走平; $\theta>\theta_{M}$ (图中 $1.1 \pi 、 1.2 \pi$ ), 壁厚 $t$ 随着渐开线展角变化 依次为增大、减小、再增大、走平; $\theta=\theta_{M}($ 图中 $\pi$ ) 时, 壁厚 $t$ 随着渐开线展角变化依次为增大、减小、 走平。

此外, $\theta$ 从 $0.8 \pi$ 增加至 $\pi$, 壁厚最小值 $t_{\min }$ 逐渐 增大, 壁厚最大值 $t_{\max }$ 逐渐减小; $\theta$ 从 $\pi$ 增加至 $1.2 \pi$, 壁厚最小值 $t_{\min }$ 逐渐减小，壁厚最大值 $t_{\max }$ 逐渐增 大。中值 $\theta_{M}=\pi$ 对应的壁厚最小值 $t_{\text {min }}$ 最大, 壁厚最 大值 $t_{\max }$ 最小。

综上，中值 $\theta_{M}=\pi$ 对应的壁厚几何性能最佳。

\section{2 控制系数 $\theta$ 对面积利用率 $\eta$ 的影响}

涡旋齿的面积利用率 $\eta$ 定义为: 浴旋齿投影面 积与终端渐开角对应的圆盘面积的比值, 如图 $8 \mathrm{a}$ 所示。 $\eta$ 越小, 表示浴旋齿用料越少, 越经济。 $\theta$ 从 $0.7 \pi$ 增加至 $1.2 \pi$ 对应的面积利用率如图 $8 b$ 所示。

$$
\eta=\frac{4 S_{c}}{\pi D_{\min }^{2}}
$$

式中, $S_{c}$ 为浴旋齿投影面积; $D_{\min }$ 为浴旋盘最小 直径。 


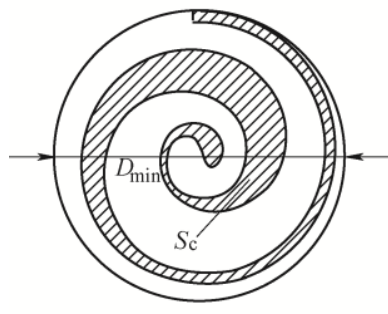

(a)

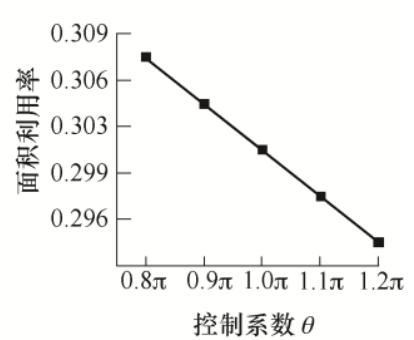

(b)
图 8 面积利用率及其变化

从图 $8 \mathrm{~b}$ 可知: $\theta$ 从 $0.8 \pi$ 增加至 $1.2 \pi, \eta$ 逐渐减 小, 但相差极小, 最大值与最小值仅相差 $1.4 \%$ 。在 实际设计中, 可忽略不计。

\section{3 控制系数 $\theta$ 对工作腔的影响}

(1) 吸气腔

$\varphi_{\text {end }}-\left(\varphi^{*}+2 n_{1} \pi\right) \geqslant 3 \pi$, 任意工作时刻的吸气腔 全部由圆渐开线 I 构成, $\theta$ 取值的变化不会对吸气 腔产生影响。

$\varphi_{\text {end }}-\left(\varphi^{*}+2 n_{1} \pi\right) \leqslant 3 \pi$ ，根据工作时刻的不同， 吸气腔的构成有两种情况: 一种是全部是基圆渐开 线 I ; 另一种是包含圆渐开线 I 和圆渐开线 II, $\theta$ 取 值的变化会对吸气腔产生影响。

(2) 压缩腔

$\varphi_{\text {end }}-\left(\varphi^{*}+2 n_{1} \pi\right) \geqslant 3 \pi+2 N \pi$ ( $N$ 为正整数), $\theta$ 取值的变化不会对第 $N$ 压缩腔产生影响。

$\varphi_{\text {end }}-\left(\varphi^{*}+2 n \pi\right) \leqslant 3 \pi+2 N \pi, \theta$ 取值的变化会 对第 $N$ 压缩腔产生影响。特别注意的是, 根据所开 的排气角 $\varphi_{p} 、 \varphi^{*}$ 取值及齿头修正的不同, $\theta$ 取值的 变化可能会对第 $N_{\text {max }}$ 压缩腔可能影响。

$\varphi^{*}-\varphi_{p} \geqslant 2 \pi, \theta$ 取值的变化不会对第 $N_{\text {max }}$ 压 缩腔产生影响; $\varphi^{*}-\varphi_{p} \leqslant 2 \pi, \theta$ 取值的变化会对 第 $N_{\text {max }}$ 压缩腔产生影响。

(3) 排气腔

$\varphi^{*} \geqslant 2 \pi$, 任意工作时刻的排气腔全部由圆渐 开线 I 、修正型线构成, $\theta$ 取值的变化不会对排气 腔产生影响。

$\varphi^{*} \leqslant 2 \pi$ ，根据工作时刻的不同，排气腔的构 成有两种情况: 一种是由圆渐开线 I、修正型线组 成; 另一种是由圆渐开线 I 、圆渐开线 II 和修正型 线组成, $\theta$ 取值的变化会对排气腔产生影响。因为 排气过程是近似恒压过程, $\theta$ 取值的变化产生的影 响可忽略不计。

以表 1 、表 2 参数值作图, 吸气腔、第一压缩腔、 第二压缩腔、排气腔对应的投影面积变化如图 9 所示。
从图 9 可知: 主轴转角小于 $180^{\circ}, 5$ 条曲线重 叠, 即吸气腔投影面积相同。主轴转角位于 $\left(180^{\circ}\right.$, $\left.900^{\circ}\right)$ 时, 5 条曲线逐渐分化, 即工作腔投影面积 逐渐分化, 但分化不大。吸气完成时刻 ( $\left.360^{\circ}\right)$ 对 应的投影面积有 $1.2 \pi>1.1 \pi>\pi>0.9 \pi>0.8 \pi$ ；压 缩终了时刻 $\left(900^{\circ}\right)$ 对应的投影面积相等。仅从此 点考虑, 控制系数 $\theta$ 越大越好。主轴转角大于 $900^{\circ}$, 5 条曲线再一次重叠，即排气腔投影面积相同。

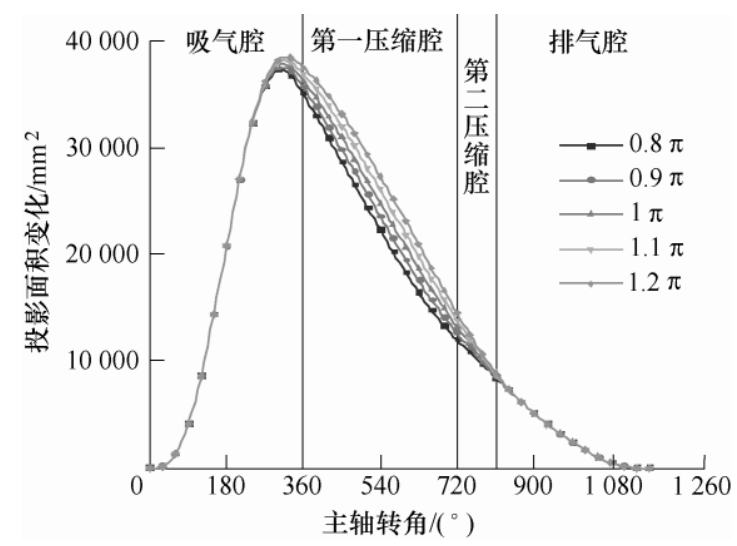

图 9 工作腔投影面积变化

\section{4 控制系数 $\theta$ 对压缩比 $\zeta$ 的影响}

压缩比 $\zeta$ 定义: 吸气完成时刻工作腔容积与压 缩终了时刻工作腔容积的比值。

$$
\zeta=\left(\frac{V_{s}}{V_{e}}\right)^{\kappa}
$$

式中, $V_{s}$ 为吸气完成时刻工作腔容积, $V_{e}$ 为压缩终 了时刻工作腔容积, $\kappa$ 为气体的等熵指数, 取 $\kappa=1$ 。

以表 1 、表 2 参数值作图, 压缩比 $\zeta$ 随 $\theta$ 的变化 如图 10a 所示。

从图 $10 \mathrm{a}$ 可知: $\theta$ 从 $0.7 \pi$ 增加至 $1.2 \pi$, 压缩比 轻微递增, 幅度极小。在实际设计中, 可忽略不计。

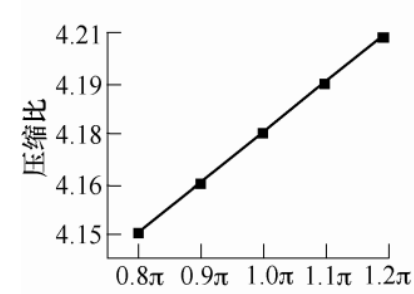

(a)

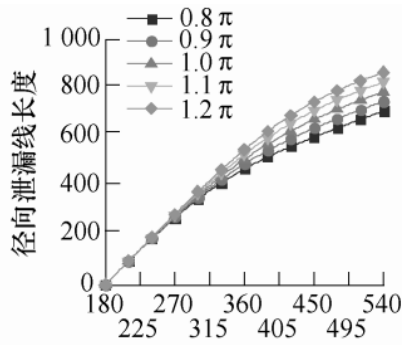

主轴转角 $/\left(^{\circ}\right)$
图 10 缩比及径向泄漏线长度

\section{5 控制系数 $\theta$ 对泄漏线长度的影响}

理想情况下，浴旋压缩机的动静浴旋齿完美啮 合, 不存在间隙。但在实际工作工程中, 涡旋压缩 
机整个工作状态均存在泄漏 ${ }^{[24]}$ 。泄漏分为 2 种: 切 向泄漏和径向泄漏。通过径向间隙产生的切向泄漏, 泄漏线长度为浴旋齿齿高。通过轴向间隙产生的径 向泄漏, 泄漏线长度为浴旋基线长度。径向泄漏的 泄漏量远远大于切向泄漏的泄漏量, 泄漏线长度越 长, 泄漏量越大。

$$
L=\int_{\varphi_{1}}^{\varphi_{2}} \sqrt{x^{\prime 2}(t)+y^{\prime 2}(t)} \mathrm{d} \varphi
$$

以表 1 、表 2 参数值作图, 变截面对应的径向 泄漏线长度 $L$ 随 $\theta$ 的变化如图 10b 所示。

从图 $10 b$ 可知: $\theta$ 从 $0.7 \pi$ 增加至 $1.2 \pi$, 径向泄 漏线长度 $L$ 逐渐增加。 $\theta$ 取 $0.7 \pi$ 和 $1.2 \pi$ 在末端对应 的径向泄漏线长度 $L$ 相差 $17.35 \%$ 。在实际设计时, 需考虑径向泄漏线长度对泄漏的影响, 尽量取小值。

综上所述，在实际应用中，控制系数 $\theta$ 取值以 中值 $\theta_{M}$ 附近对应的几何性能较优。

\section{3 控制系数 $\varphi^{*}$ 对几何性能的影响}

其他参数保持不变, 仅改变控制系数 $\varphi^{*}$, 研究 $\varphi^{*}$ 取值的变化对几何性能的影响。

以表 1 参数值作图, 仅改变 $\varphi^{*}$ 的取值, 其它参 数不变(注: 中值 $\theta_{M}$ 与 $\varphi^{*}$ 有关, 随之改变), $\varphi^{*} 、 \theta_{M}$ 的取值如表 3, 生成的一系列基线如图 $5 \mathrm{~b}$ 所示。

\section{表 $3 \varphi^{*} 、 \theta_{M}$ 取值表}

\begin{tabular}{ccc}
\hline 数值 & 渐开角 $\varphi^{*} / \mathrm{rad}$ & 中值 $\theta_{M} / \mathrm{rad}$ \\
\hline 数值 1 & $1.5 \pi$ & $0.75 \pi$ \\
数值 2 & $2 \pi$ & $\pi$ \\
数 3 & $2.5 \pi$ & $1.25 \pi$ \\
数值 4 & $3 \pi$ & $1.5 \pi$ \\
\hline
\end{tabular}

\section{1 控制系数 $\varphi^{*}$ 对涡旋齿壁厚 $t$ 的影响}

以表 1 、表 3 参数值作图, 生成的壁厚 $t$ 随渐开 线展角变化如图 11 所示。

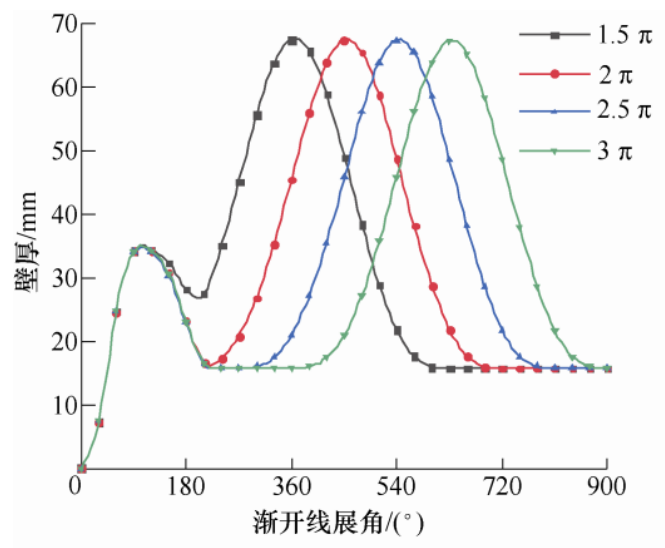

图 11 壁厚随渐开线展角的变化
从图 11 可知: $\varphi^{*}$ 取 $1.5 \pi 、 2 \pi 、 2.5 \pi 、 3 \pi$ 对应 的壁厚 $t$ 变化规律均为先增大后减小, 最后走平(除 去齿头修正部分)，仅初值和终值不同; 对应的最大 壁厚值 $t_{\text {max }}$ 和最小壁厚值 $t_{\text {min }}$ 均相同。控制系数 $\varphi^{*}$ 的 变化对浴旋齿壁厚 $t$ 仅影响起始位置, 对其它无影 响。在实际设计中, 可忽略不计。

\section{2 控制系数 $\varphi^{*}$ 对面积利用率 $\boldsymbol{\eta}$ 的影响}

以表 1 、表 3 参数值作图, 面积利用率 $\eta$ 随 $\varphi^{*}$ 的 变化如图 12a 所示。

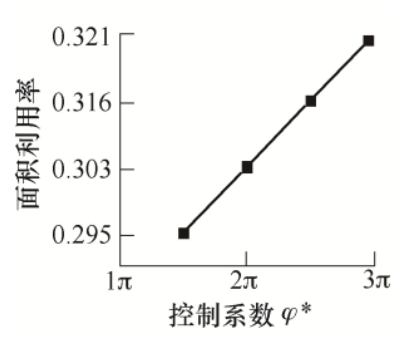

(a)

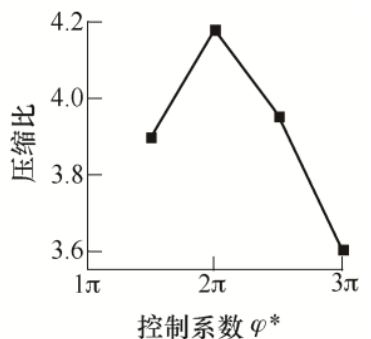

(b)
图 12 面积利用率及压缩比

从图 $12 \mathrm{a}$ 可知: $\varphi^{*}$ 从 $1.5 \pi$ 增加至 $3 \pi, \eta$ 逐渐增 大, 但相差较小, 最大值与最小值仅相差 $2.6 \%$ 。在 实际设计中, 可忽略不计。

\section{3 控制系数 $\varphi^{*}$ 对工作腔的影响}

关于控制系数 $\varphi^{*}$ 对工作腔的影响, 与第 2.3 节 类似, 这里不做详细说明。

以表 1、表 3 参数值作图, 吸气腔、第一压缩 腔、第二压缩腔、排气腔对应的投影面积变化如图 13 所示。

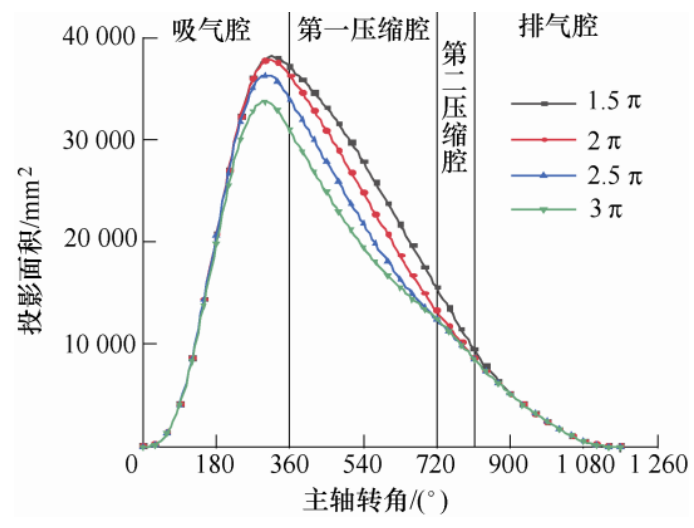

图 13 工作腔投影面积变化

从图 13 可知: 吸气完成时刻 $\left(360^{\circ}\right)$ 对应的投影 面积有 $1.5 \pi>2 \pi>2.5 \pi>3 \pi$; 压缩终了时刻 $\left(900^{\circ}\right)$ 对应的投影面积有 $1.5 \pi>2 \pi=2.5 \pi=3 \pi$ 。仅从此点考 虑, 控制系数 $\varphi^{*}$ 取 $2 \pi$ 优于 $2.5 \pi, 2.5 \pi$ 优于 $3 \pi, \varphi^{*}$ 取 $1.5 \pi$ 与 $2 \pi$ 的优劣无法比较。 


\section{4 控制系数 $\varphi^{*}$ 对压缩比 $\zeta$ 的影响}

以表 1 、表 3 参数值作图, 压缩比 $\zeta$ 随 $\varphi^{*}$ 的变 化如图 $12 b$ 所示。

从图 $12 \mathrm{~b}$ 可知: 从压缩比考虑, 控制系数 $\varphi^{*}$ 取 $2 \pi$ 最优, 其余依次为 $2.5 \pi 、 1.5 \pi 、 3 \pi$ 。控制系数 $\varphi^{*}$ 取 $2 \pi$ 对应的吸气腔一半由圆渐开线 I 组成, 一半由圆 渐开线 II 组成。此时, $\varphi^{*}$ 与 $\varphi_{\text {end }}$ 存在以下关系

$$
\varphi^{*}=\varphi_{\text {end }}-2\left(n_{1}+1\right) \pi
$$

在设计时考虑此点, 有利于设计出高压比的涡 旋齿。

\section{5 控制系数 $\varphi^{*}$ 对泄漏线长度的影响}

以表 1 、表 3 参数值作图, 变截面对应的径向 泄漏线长度 $L$ 随 $\varphi^{*}$ 的变化如图 14 所示。

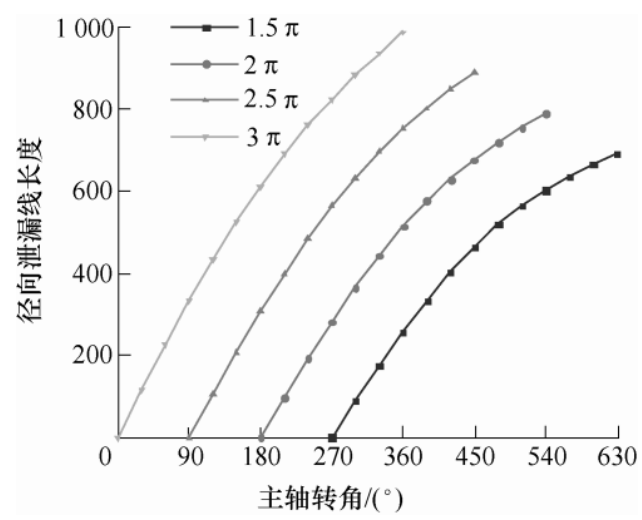

图 14 径向泄漏线长度

从图 14 可知: $\varphi^{*}$ 取 $1.5 \pi 、 2 \pi 、 2.5 \pi 、 3 \pi$ 时变截 面径向泄漏线起始位置对应的主轴转角分别为 $270^{\circ} 、 180^{\circ} 、 90^{\circ} 、 0^{\circ}$, 终止位置对应的主轴转角分 别为 $630^{\circ} 、 540^{\circ} 、 450^{\circ} 、 360^{\circ}$ 。 $\varphi^{*}$ 取 $3 \pi$ 与 $1.5 \pi$ 在末 端对应的径向泄漏线长度 $L$ 相差 $42.85 \%$ 。在设计 时, 需考虑径向泄漏线长度对泄漏的影响, 尽量取 小值。

综上所述，在实际应用中，控制系数 $\varphi^{*}$ 取值以 $\varphi^{*}=\varphi_{\text {end }}-2\left(n_{1}+1\right) \pi$ 附近对应的几何性能较优。

\section{4 控制系数 $R_{o r}$ 对几何性能的影响}

其他参数保持不变, 仅改变控制系数 $R_{o r}$, 研究 $R_{o r}$ 取值的变化对几何性能的影响。

以表 1 参数值作图, 仅改变 $R_{o r}$ 的取值, 其它 参数不变, $R_{o r}$ 的取值如表 4 , 生成的一系列基线如 图 1 所示。因基线无变化，对应的径向泄漏线长度 无变化，此小节对泄漏线长度不做讨论。
表 $4 \boldsymbol{R}_{o r}$ 取值表

\begin{tabular}{cc}
\hline 回转半径 $R_{o r} / \mathrm{mm}$ & 数值 \\
\hline 数值 1 & $3 \pi$ \\
数值 2 & $4 \pi$ \\
数值 3 & $5 \pi$ \\
数值 4 & $6 \pi$ \\
数值 5 & $7 \pi$ \\
\hline
\end{tabular}

\section{1 控制系数 $\boldsymbol{R}_{o r}$ 对涡旋齿壁厚 $\boldsymbol{t}$ 的影响}

以表 1 、表 4 参数值作图, 生成的壁厚 $t$ 随渐开 线展角变化如图 15 所示。（注：此小节为满足修正 要求，其修正角与 2、3 小节修正角不同）

从图 15 可知: $R_{o r}$ 取 $3 \pi 、 4 \pi 、 5 \pi 、 6 \pi 、 7 \pi$ 对应 的壁厚 $t$ 变化规律均为先增大后减小，最后走平(除 去齿头修正部分), 对应的最大壁厚值 $t_{\max }$ 和最小壁 厚值 $t_{\mathrm{min}}$ 不同。任意渐开线展角对应的壁厚 $t$, 均有 $3 \pi>4 \pi>5 \pi>6 \pi>7 \pi$ 。在满足强度条件时, 壁厚 $t$ 取 偏小值较好。太薄不易加工, 太厚经济性低。在实 际设计中, 需考虑此点。

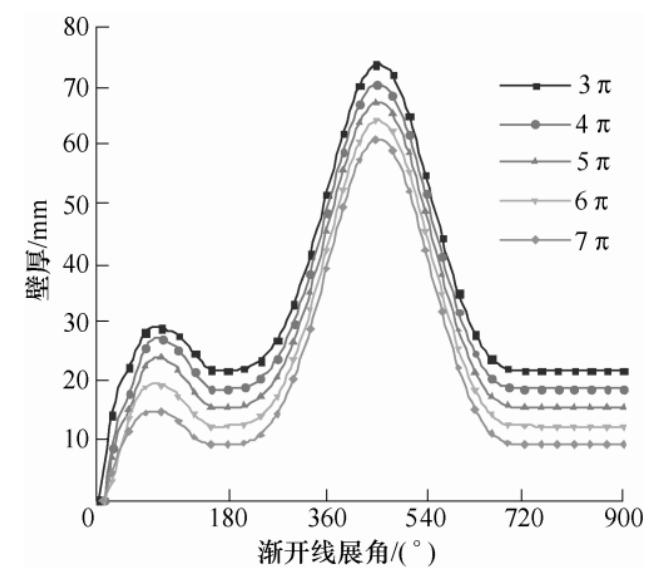

图 15 壁厚随渐开线展角的变化

\section{2 控制系数 $\boldsymbol{R}_{o r}$ 对面积利用率 $\boldsymbol{\eta}$ 的影响}

以表 1 、表 4 参数值作图, 面积利用率 $\eta$ 随 $\varphi^{*}$ 的 变化如图 16a 所示。

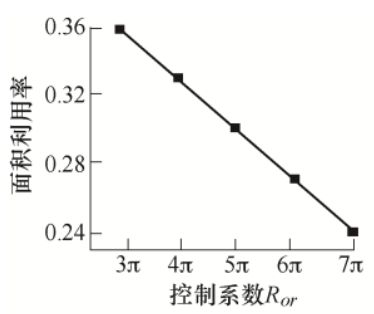

(a)

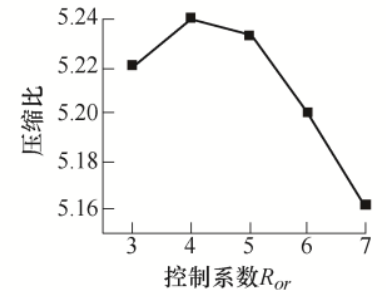

(b)
图 16 面积利用率及压缩比

从图 16a 可知: $R_{o r}$ 从 $3 \pi$ 增加至 $7 \pi, \eta$ 逐渐减小, 且相差较大, 最大值与最小值相差 $11.59 \%$ 。在实际 设计中，尽量取小值，节省成本。 


\section{3 控制系数 $R_{o r}$ 对工作腔的影响}

关于控制系数 $R_{o r}$ 对工作腔的影响, 与 2.3 节类 似, 这里不做详细说明。

以表 1、表 4 参数值作图, 吸气腔、第一压缩 腔、第二压缩腔、排气腔对应的投影面积变化如 图 17 所示。

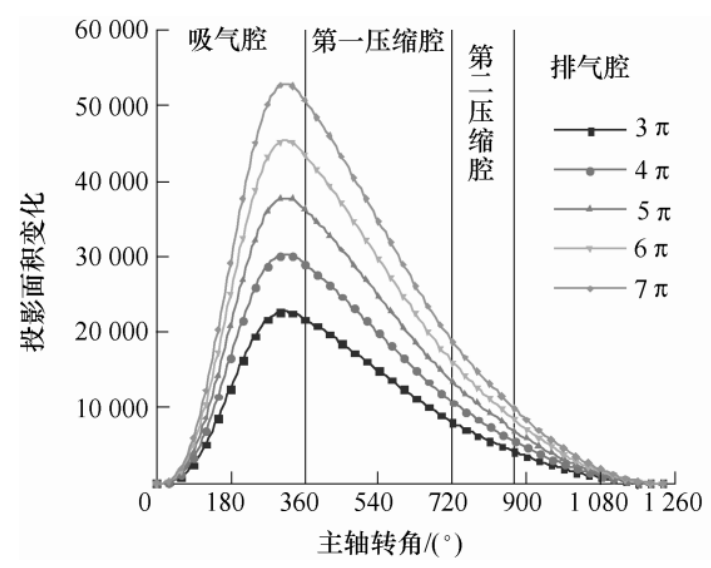

图 17 工作腔投影面积变化

从图 17 可知: 在任意主轴转角对应的投影面 积, 均有 $7 \pi>6 \pi>5 \pi>4 \pi>3 \pi$ 。随着控制系数 $R_{o r}$ 逐 渐增大, 投影面积大幅度增大, 即吸气量大幅度提 高, 单位时间内排气量大幅度提高。从此点考虑, 在满足其他设计要求时, 控制系数 $R_{o r}$ 尽量取较 大值。

\section{4 控制系数 $\boldsymbol{R}_{o r}$ 对压缩比 $\zeta$ 的影响}

以表 1 、表 4 参数值作图, 压缩比 $\zeta$ 随 $R_{o r}$ 的变 化如图 16b 所示。

从图 $16 \mathrm{~b}$ 可知: 从压缩比考虑, 控制系数 $R_{o r}$ 取 $4 \pi$ 最优, 其余依次为 $5 \pi 、 3 \pi 、 6 \pi 、 7 \pi$, 但最大 值与最小值相差极小, 仅相差 $1.5 \%$ 。在实际设计中, 可忽略不计。

综上所述，在实际应用中，在满足受力特性、 运动特性等的条件下, 控制系数 $R_{o r}$ 取值尽量取较 大值，几何性能较优。

\section{5 两类几何模型对比}

以圆渐开线 I 为基础, 构建圆渐开线 $I+$ 高次曲 线+圆渐开线 I 的变截面浴旋压缩机几何模型, 结合 上文建立的圆渐开线 I +圆渐开线 II +圆渐开线 I 的变截面压缩机几何模型, 对比分析两类变截面浴 旋压缩机的各项几何性能。

\section{1 高次曲线基线方程及内外壁方程}

$$
\left[\begin{array}{l}
X \\
Y
\end{array}\right]=R_{g}\left[\begin{array}{l}
\cos \varphi \\
\sin \varphi
\end{array}\right]+R_{S}\left[\begin{array}{l}
\sin \varphi \\
-\cos \varphi
\end{array}\right]
$$

式中, $\varphi \in\left[\varphi^{*}, \varphi^{*}+2 n_{2} \pi\right] ; R_{\mathrm{g}} 、 R_{s}$ 分别为基圆半径、 展弦，计算公式如下

$$
\left[\begin{array}{l}
R_{g} \\
R_{s}
\end{array}\right]=\left[\begin{array}{l}
\left\{\begin{array}{l}
C_{2}+2 C_{3}(\varphi-0.5 \pi) \\
+3 C_{4}(\varphi-0.5 \pi)^{2}
\end{array}\right\} \\
\left\{\begin{array}{l}
C_{1}+C_{2}(\varphi-0.5 \pi)+C_{3}(\varphi- \\
0.5 \pi)^{2}+C_{4}(\varphi-0.5 \pi)^{3}
\end{array}\right\}
\end{array}\right\}
$$

式中, $C_{1} 、 C_{2} 、 C_{3} 、 C_{4}$ 为待定系数, 根据所选参数 $\varphi^{*} 、 n_{1} 、 n_{2}$ 确定。以表 1 参数值求解待定系数值, 如表 5 所示。

表 5 待定系数值

\begin{tabular}{cc}
\hline 待定系数值 & 数值 \\
\hline$C_{1}$ & 174.7510 \\
$C_{2}$ & -68.75000 \\
$C_{3}$ & 11.93662 \\
$C_{4}$ & 0.50661 \\
\hline
\end{tabular}

通过法向等距法生成内外壁，方程如下。

涡旋齿内壁方程

$$
\begin{aligned}
& {\left[\begin{array}{l}
X \\
Y
\end{array}\right]=R_{g}\left[\begin{array}{l}
\cos \varphi \\
\sin \varphi
\end{array}\right]+R_{S}\left[\begin{array}{l}
\sin \varphi \\
-\cos \varphi
\end{array}\right]+} \\
& {\left[\begin{array}{l}
0.5 R_{o r} \sin \varphi \\
-0.5 R_{o r} \cos \varphi
\end{array}\right]}
\end{aligned}
$$

式中, $\varphi \in\left[\varphi^{*}, \varphi^{*}+2 n_{2} \pi\right]$ 。

涡旋齿外壁方程

$$
\begin{aligned}
& {\left[\begin{array}{l}
X \\
Y
\end{array}\right]=R_{g}\left[\begin{array}{l}
\cos \varphi \\
\sin \varphi
\end{array}\right]+R_{S}\left[\begin{array}{l}
\sin \varphi \\
-\cos \varphi
\end{array}\right]-} \\
& {\left[\begin{array}{l}
0.5 R_{o r} \sin \varphi \\
-0.5 R_{o r} \cos \varphi
\end{array}\right]}
\end{aligned}
$$

式中, $\varphi \in\left[\varphi^{*}, \varphi^{*}+2 n_{2} \pi\right]$ 。

\section{2 基线及内外壁型线对比}

以表 1 、表 5 参数值作图，圆渐开线 I+高次曲 线+圆渐开线 I 和圆渐开线 I +圆渐开线 II +圆渐开 线 I 的基线如图 18a 所示。

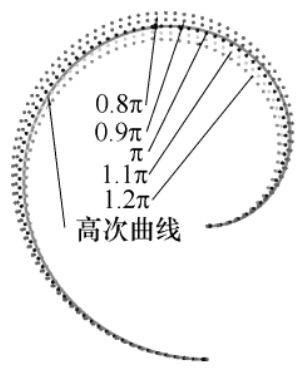

(a)

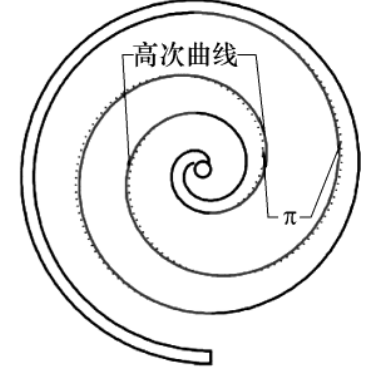

(b)
图 18 基线及内外壁型线 
从图 18a 可知, 高次曲线起点部分位于圆渐开 线 II 的外侧, 高次曲线终点部分位于圆渐开线 II 的 内侧。高次曲线与 $\theta=\pi$ (即中值 $\theta_{M}$ ) 的圆渐开线 II 最为相似，生成的内外壁型线如图 18b 所示。

从图 $18 \mathrm{~b}$ 可知, 高次曲线与 $\theta=\pi$ 的圆渐开线 II 生成的内外壁型线相似, 但有细微差别。因此, 其各项几何性能有些差别。

\section{3 各项几何性能对比}

取高次曲线和 $\theta=\pi$ 的圆渐开线 II 建立的几何 模型进行分析，壁厚 $t$ 、面积利用率 $\eta$ 和压缩比 $\zeta$ 、 工作腔变化、径向泄漏线长度 $L$ 对比, 分别如图 19a、 19b、19c、19d 所示。

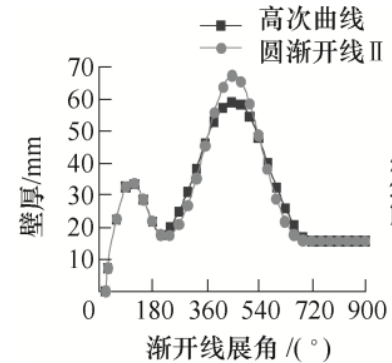

(a)

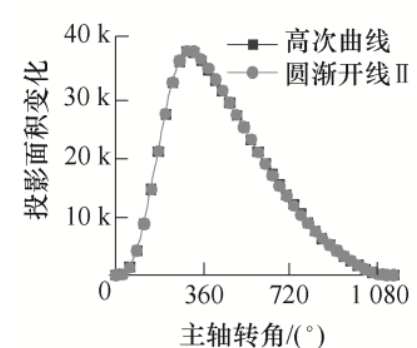

(c)

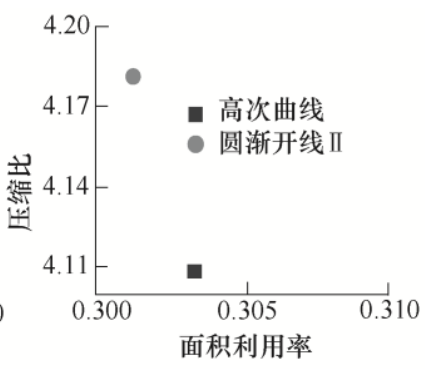

(b)

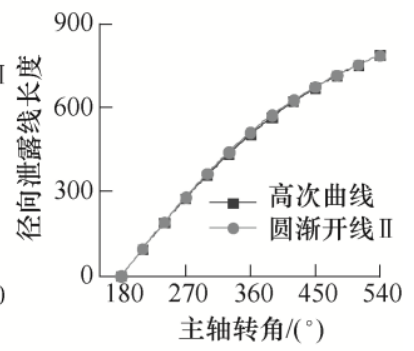

(d)
图 19 各项几何性能对比

从图 19a 可知, 变截面壁厚起始和终止位置附 近，圆渐开线 II 对应的壁厚 $t$ 大于高次曲线对应的 壁厚 $t$; 在中间段，高次曲线对应的壁厚 $t$ 大于圆渐

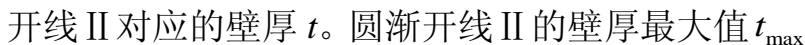
大于高次曲线对应的壁厚最大值 $t_{\text {max }}$; 两者的壁厚 最小值 $t_{\min }$ 基本相等。因此, 关于壁厚几何性能, 高次曲线优于圆渐开线 II 。

从图 $19 \mathrm{~b}$ 可知, 关于面积利用率 $\eta$, 圆渐开线 II 小于高次曲线, 性能更优; 关于压缩比 $\zeta$, 圆渐 开线 II 大于高次曲线。此两点的几何性能, 圆渐开 线 II 优于高次曲线。

从图 19c、图 19d 可知, 关于投影面积变化和 变截面径向泄漏线长度, 两者变化规律基本相同。 此两点的几何性能，可认为基本相同。

综上，两类变截面浴旋压缩机几何模型各有优 劣, 圆渐开线 $\mathrm{I}+$ 高次曲线+圆渐开线 $\mathrm{I}$ 的变截面浴
旋压缩机几何模型与 $\theta_{M}$ 对应的圆渐开线 I+圆渐开 线 II+圆渐开线 I 的变截面压缩机几何模型相似, 在 实际应用可相互替代。因此, 可认为高次曲线变截 面涡旋压缩机是一系列圆渐开线变截面浴旋压缩机 中的一种，任意高次曲线变截面涡旋压缩机均可由 圆渐开线变截面浴旋压缩机替代。

\section{6 结论}

(1) 提出一种由不同基圆半径的圆渐开线组成 的新型变截面浴旋压缩机型线, 组成形式为圆渐开 线 I +圆渐开线 II+圆渐开线 I 。论述型线的生成方 法, 给出型线的一般方程。

(2) 建立一系列圆渐开线变截面浴旋压缩机的 几何模型, 分析控制系数 $\theta 、 \varphi^{*} 、 R_{o r}$ 对变截面浴 旋压缩机几何性能的影响。在实际应用中, 控制系 数 $\theta$ 取值以中值 $\theta_{M}$ 附近对应的几何性能较优，控制 系数 $\varphi^{*}$ 取值以 $\varphi^{*}=\varphi_{\text {end }}-2\left(n_{1}+1\right) \pi$ 附近对应的几何 性能较优, 控制系数 $R_{o r}$ 尽量取较大值, 几何性能 较优。

(3) 对比分析两类变截面浴旋压缩机的各项几 何性能, 高次曲线变截面浴旋压缩机与中值 $\theta_{M}$ 对应 的圆渐开线变截面浴旋压缩机相似，可相互替代。 可认为高次曲线变截面浴旋压缩机是一系列圆渐开 线变截面浴旋压缩机中的一种，任意高次曲线变截 面涡旋压缩机均可由圆渐开线变截面浴旋压缩机 替代。

\section{参 考 文 献}

[1] PENG B, ZHU B G, VINCENT L. Theoretical and experimental analysis of scroll expander[C]// 23rd International Compressor Engineering Conference at Purdue, July 11-14, 2016, 1334: 1-12.

[2] IVIEV V I, MISYURIN S Y. Calculated and experimental characteristics of a scroll machine operating in the air moter mode[J]. Doklady Physics, 2017，62(1): 42-45.

[3] LU Y, ROSKILLY A P, JIANG L, et al. Analysis of a $1 \mathrm{kw}$ organic ranking cycle using a scroll expander for engine coolant and exhaust heat recovery[J]. Frontiers in Energy, 2017, 11(4): 527-534.

[4] DONG A C, KWON O K, OH M D. An experimental study on semiconductor process chiller using the digital scroll compressor[J]. Journal of Mechanical Science and Technology, 2014, 28(8): 3345-3352.

[5] 彭斌, 李要红, 赵生显. 浴旋膨胀机的性能模拟 [J].中 
国机械工程, 2018，29(8): 965-970， 978.

PENG Bin, LI Yaohong, ZHAO Shengxian. Performance simulation for scroll expanders[J]. China Mechanical Engineering, 2018, 29(8): 965-970, 978.

[6] BRYCE R S, ECKHARD A G. Variable wall thickness Scroll geometry modeling with use of a control volume approach[J]. International Journal of Refrigeration, 2013, 36(7): 1809-1820.

[7] 李连生. 线段渐开线浴旋压缩机的几何理论[J]. 流体机 械, 1994, 22(12): 22-28.

LI Liansheng. Geometric theory of line involute scroll compressor[J]. Fluid Machinery, 1994，22(12): 22-28.

[8] BELL I. Theoretical and experimental analysis of liquid flooded compression in scroll compressors[D]. West Lafayette: Purdue University, 2011.

[9] MORISHITA E, SUGIHARA M, INABA T, et al. Scroll compressor analytical model[C]//Proceedings of International Compressor Engineering Conference, January, 1984, Purdue University, West Lafayette, Indiana, USA. Indiana: Ray W, Herrick Laboratories Press, 1984: 487-495.

[10] 王立存, 李世六, 明勇, 等. 浴旋压缩机型线耦合机理 动态性研究 [J]. 哈尔滨工业大学学报, 2009, 41(7): $11-15$.

WANG Licun, LI Shiliu, MING Yong, et al. Dynamics of joggling principle for scroll profiles of scroll compressor[J]. Journal of Harbin Institute of Technology, 2009, 41(7): 11-15.

[11] 彭斌, 张朋成. 变截面浴旋膨胀机的数学模型及试验 研究[J]. 机械设计与制造工程, 2018, 47(07): 115-120. PENG Bin, ZHANG Pengcheng. Mathematical model and experimental study of a variable thickness scroll expander[J]. Machine Design and Manufacturing Engineering, 2018, 47(7): 115-120.

[12] 彭斌, 孙迎, 张力. 变截面涡旋压缩机几何模型及摩 擦损失模型 $[J]$. 兰州理工大学学报, 2012, 38(3): 34-38. PENG Bin, SUN Ying, ZHANG Li. Geometrical and frictional loss model for scroll compressor with variable scroll thickness[J]. Journal of Lanzhou University of Technology, 2012, 38(03): 34-38.

[13] 彭斌, 张朋成. 一种变截面涡旋膨胀机的几何模型及 容积变化[J]. 机械设计与制造, 2019(6): 5-8.

PENG Bin, ZHANG Pengcheng. Geometric model and volume change of a variable cross section scroll expander[J]. Machinery Design\&Manufacture, 2019(6): 5-8.
[14] BUSH J W, BEAGLE W P, HOUSMEN M E. Maximizing scroll compressor displacement using generalized wrap geometry[C]//Proceeding of the International Compressor Engineering Conference at Purdue, USA, 1994: 205-210.

[15] 陈进, 张永栋, 宋立权, 等. 基于多目标遗传算法的 浴旋型线形状优化 $[\mathrm{J}]$. 机械工程学报, 2005, 41(1): 172-175.

CHEN Jin, ZHANG Yongdong, SONG Liquan, et al. Profile optimization of scrolls based on multiobjective genetic algorithms[J]. Journal of Mechanical Engineering, 2005, 41(1): 172-175.

[16] 彭斌, 孙迎. 变截面浴旋压缩机数学模型及试验研究 [J]. 机械工程学报, 2015，51(14): 185-191.

PENG Bin, SUN Ying. Investigation of mathematical modeling and experiment for variable thickness scroll compressor[J]. Journal of Mechanical Engineering, 2015, 51(14): 185-191.

[17] MURRY C. Variable wall thickness boosts scroll compressor's performance[J]. Design News, 1997, 53(5): 116-117.

[18] 刘涛, 鸟再新, 刘振全. 法向等距线法生成浴旋压缩机 型线的研究[J]. 机械工程学报, 2004, 40(6): 55-58.

LIU Tao, WU Zaixin, LIU Zhenquan. Study on generating profile with nomal-equidistant-curve method for scroll compressor[J]. Journal of Mechanical Engineering, 2004, 40(6): 55-58.

[19] 王君, 刘振全. 浴旋压缩机渐开线类型线的双圆弧修正 [J]. 机械工程学报, 2005, 41(9): 202-206.

WANG Jun, LIU Zhenquan. Double circle arc of involute type wrape of scroll compressor[J]. Journal of Mechanical Engineering, 2005, 41(9): 202-206.

[20] 丁佳男, 张英莉, 岳向吉, 等. 涡旋压缩机的变基圆型 线研究 [J]. 东北大学学报(自然科学版), 2019, 40(5): 722-727.

DING Jianan, ZHANG Yingli, YUE Xiangji, et al. Study on the circular profile of scroll compressors with changing radii $[\mathrm{J}]$. Journal of Northeastern University(Natural Science), 2019, 40(5): 722-727.

[21] 王国梁. 采用双圆弧加直线单元组合型线的浴旋压缩 机理论及试验研究 [J]. 机械工程学报, 2010, 46(10): 144-147.

WANG Guoliang. Theoretical and experimental research on the scroll compressor with arc-arc-line unit combined profiled[J]. Journal of Mechanical Engineering, 2010, 
46(10): 144-147.

[22] PENG B, LEMORT V, LEGROS A, et al. Variable thickness scroll compressor performance analysis-Part I: Geometric and thermodynamic modelling[J]. Proceedings of the Institution of Mechanical Engineering, 2017, 231 (4): 633-640.

[23] 王国梁. 基于对称圆弧修正的单元组合型线浴旋压缩 机特性研究[J]. 机械工程学报, 2011, 47(16): 139-142. WANG Guoliang. Investigation of wrap profile modification based on symmetric arc shaped curves for the scroll compressor with unit combined profile[J]. Journal of Mechanical Engineering, 2011, 47(16): 139-142.
[24] 彭斌, 赵生显, 李要红. 新型无油浴旋压缩机性能 [J]. 中国机械工程，2018，29(24): 2917-2924.

PENG Bin, ZHAO Shengxian, LI Yaohong. Performances of new oil-free scroll compressors[J]. China Mechanical Engineering, 2018, 29(24): 2917-2924.

作者简介: 张朋成, 男, 1990 年出生, 博士研究生。主要研究方向为浴 旋机械、低温余热发电。

E-mail: zhangpc1990@126.com

彭斌(通信作者), 男, 1976 年出生, 博士, 教授, 博士研究生导师。主 要研究方向为浴旋机械、低温余热发电、复杂机电系统的集成科学和故 障诊断。

E-mail: pengb2000@163.com 